\section{Top-Off Exercise in Washington, DC}

Masato Homma, MD; Yasubiro Otomo, MD;

Hirosbi Henmi, MD; Takasbi Arai, MD

National Disaster Medical Center, Japan

Objective: To report on a TOP-OFF exercise conducted in the Washington, DC area, and describe the differences from Japanese planning against weapons of mass destruction (WMD) attacks.

Methods: In May 2000, the government of United States conducted a large-scale exercise for responding to WMD attacks in three cities: 1) Denver, Colorado; 2) Portsmouth, New Hampshire, and the Washington, DC-area. These exercises were called "TOP-OFF" because of the involvement of top officials according to the direction of the US Congress in 1998. The exercise conducted in Washington was called "National Capital Region 2000" (NCR-2000).

Results: The NCR-2000 was a simulated bombing and radiological attack. It required detection of radio-activity, patient collection, decontamination (DECON), patient care, and transportation to hospitals by ambulances.

In Japan, on-site decontamination (DECON) has not been developed fully because: 1) It was after the attack on 11 September 2001 that the Japanese cabinet office officially announced a guideline for responding to WMD attack, and introduced the basic concept of on-site DECON by fire, police, and the military; 2) Japanese medical law does not allow paramedics to give medications or advance life support, such as intubation, and severely ill patients are transported urgently to a hospital before receiving DECON; 3) Medical teams are not fully equipped for a WMD attack; and 4) It is difficult to provide necessary medical care both at the site and inside the hospital.

Conclusion: In Japan, local core hospitals have a crucial role in dealing with contaminated patients; they have not fully equipped for fighting against WMD attacks.

Keywords: advanced life support; decontamination; exercise; hospitals; intubation; medical care; weapons of mass destruction

Prehosp Disast Med 2002;17:s30.

\section{Training Hospital Staff to Cope with a Chemical Mass Casualty Event (CMCE)}

Bianca Lederman, $M D$; Nurith Fertel, $R N, B A$

Nursing Emergency Services, Tel Aviv Sourasky Medical Center, Tel Aviv, Israel

Since chemical weapons and mass destruction events emphasized their potential catastrophic effect on the population, hospitals in Israel have been committed to be prepared for the administration of optimal medical services to a large number of casualties. Successful coping mechanisms of the medical staff for such treatment necessitates special organization, based on five phases:

1. Establishing standing orders and instructions;

2. Preparation of decontamination facilities and protective gear for the personnel;

3. Designation of the hospital staff;

4. Training programs and drills.
These drills take place on an annual basis, and simulate a realistic scenario. This requires the hospital to allocate the necessary staff and equipment, update the instructions, the standing orders and implement acquired knowledge. Videos recorded during these drills become a visual educational aid for future training.

Preparedness for a drill is a long and complicated procedure. The model contents four crucial steps:

1. Designation of the hospital staff;

2. Preparation of the special training equipment required;

3. Training the relevant staff; and

4. Training voluntary and ancillary teams who participate in the drill.

Conclusion: "War Games" - (drills) are a very important part of the training program aimed to ensure the ongoing preparedness and alert of the medical staff.

Keywords: chemical; coping; decontamination; drill; mass casualties; personal protective equipment; staff; training

Prehosp Disast Med 2002;17:s30.

\section{Night Field Drill for Disaster Medical Assistance Team (DMAT)-Full-Scale Mountain Exercise Chia-Chang Chuang; ${ }^{1}$ Cbib-Hsien Cbi; ${ }^{1}$ Hung-Jung Lin, ${ }^{2}$ Yuang-Lung Yen, ${ }^{2}$ Ming-Che Tsai ${ }^{1}$}

1. Department of Emergency Medicine, National Cheng Kung University Hospital, Tainan, Taiwan

2. Department of Emergency Medicine, Chi Mei Foundation Hospital, Tainan, Taiwan

Objective: A Disaster Medical Assistant Team (DMAT) is an emergency response team available to provide medical services following a devastating disaster. They have been developed and established in many countries including Taiwan since 2000. The experiences from a night field drill in a mountainous area were presented. It was the first large-scale DMAT exercise in Taiwan. The study compared the differences between nighttime and daytime drills in the field of deployment and preparedness.

Methods: This outside drill was held at Nan-tou county (mid-Taiwan), which was near the epicenter of Chi-Chi earthquake that struck Taiwan on 21 September 1999. It consisted of a 5-day (12-15 September 2001) course for both national DMATs and local DMATs. Four sections including operation, planning, logistics and administrative were deployed for this joint drill. Tabletop exercises and radio communication were scheduled in the training course. Mobile multiplier shelter with and electric power system was introduced and used for major illumination system.

Results: Two national DMATs and six local DMATs from northern and southern Taiwan joined this field exercise. A total of 160 persons were enrolled. The major differences at nighttime drill included: 1) more mistakes and wrong judgments were made at the triage area; 2) short of manpower; 3 ) increase in electric power; and 4) more difficulty with communication between each other in the dark area. Conclusion: The experience gained from this night, field drill was an important training for DMATs. How to overcome these inconveniences and even worse situations is another challenge for emergency response team.

Keywords: communication; DMAT; drill; electricity; experiences; 